\title{
Laryngeal Edema, CTCAE
}

National Cancer Institute

\section{Source}

National Cancer Institute. Laryngeal Edema, CT CAE. NCI Thesaurus. Code C143636.

A disorder characterized by swelling due to an excessive accumulation of fluid in the

larynx. 